Running head: TARGET WORD SEARCH IN CHINESE TEXT

\title{
Searching for a word in Chinese text: \\ Insights from eye movement behaviour
}

\author{
Xiaotong Wang ${ }^{1}$ \\ Xue Sui ${ }^{1}$ \\ Sarah J. White ${ }^{2}$ \\ ${ }^{1}$ Institute of Psychology, Liaoning Normal University, China \\ ${ }^{2}$ Department of Neuroscience, Psychology and Behaviour, University of Leicester, UK
}

Co-corresponding Authors:

Sarah J. White, Department of Neuroscience, Psychology and Behaviour, University of Leicester, George Davies Centre, University Road, Leicester, LE1 7HA, UK. s.j.white@le.ac.uk

Xue Sui, Institute of Psychology, Liaoning Normal University, 850 Huanghe Road, Shahekou district, Dalian, 116029, China. suixue@lnnu.edu.cn

Word count excluding References, Tables and Figure Captions: 6142 


\begin{abstract}
Locating relevant information in text is an important aspect of the reading process, however relatively few studies have examined this, especially for logographic languages such as Chinese. The present study examines eye movement behaviour during search for a target word in Chinese sentences, compared with reading the sentences for comprehension. Although there were clear effects of word frequency during reading for comprehension, the study shows no evidence for an influence of the word frequency of non-target words on eye movement behaviour during target word search. The results are in line with previous research undertaken in English (Rayner \& Fischer, 1996), such that during search for a target word, eye movement behaviour for non-target words is largely driven by superficial processing of those words. The study also highlights the prevalence of word skipping, indicating that words are often sampled only in visually degraded parafoveal vision during target word search in Chinese.
\end{abstract}

Keywords: Reading, Search, Chinese, Word frequency, Eye movements 


\section{Introduction}

Considerable research has been undertaken into eye movement behaviour during reading, both for alphabetic languages and for reading in Chinese (for reviews see: Rayner, 2009; Zang, Liversedge, Bai, \& Yan, 2011). However this work has focused on understanding the mechanisms underlying reading for comprehension and relatively few studies have focused on how we seek and comprehend relevant information within text (e.g. Kaakinen, Hyönä, \& Keenan, 2002). Understanding the mechanisms that underlie search for specific information is especially important now that so much textual information is available (Liu, 2005), such that locating relevant information is vital to the everyday reading process. Revealing the mechanisms that underlie the process of searching for words within text may ultimately have implications for optimising text displays (e.g. Scialfa, Caird, Connolly, \& Cosmescu, 1998). Some types of search, such as search for relevant topics (White, Warrington, McGowan, \& Paterson, 2015), related words (Léger, Rouet, Ros, \&Vibert, 2012) or answers to questions (Cauchard, Eyrolle, Cellier, \& Hyona, 2010; Klusewitz \& Lorch, 2000) necessarily involve linguistic processing of the text. In contrast, the present study focuses on the mechanisms underlying the process of searching for a specific target word, which may be achieved with only superficial processing of the text. The current study examines search for a target word within Chinese text, and the procedure is similar to Rayner and Fischer's (1996) classic study in English. In line with this original study, the current work examines search for a target word within a series of single sentences.

A few studies have previously examined eye movement behaviour during search for specific target words within meaningful text in English (Pollatsek, Raney, LaGasse, \& 
Rayner, 1993; Rayner \& Fischer, 1996; Rayner \& Raney, 1996; Spragins, Lefton, \& Fisher, 1976). Other studies have examined eye movements during search for words within lists or within a sequence of presented words (Dampuré, Ros, Rouet, \& Vibert, 2012; Léger et al., 2012; Ojanpää, Näsänen, \& Kojo, 2002). A few previous studies have examined eye movement behaviour during search for a Chinese character, both within arrays of Chinese characters (Goonetilleke, Lau, \& Smith, 2002; Rayner, Li, Williams, Cave, \& Well, 2007; Shen \& Reingold, 2001; see also Zhou, Mo, Zhang, \& Ding, 2017) and within passages of Chinese text, counting occurrences of a particular character (Rayner et al., 2007; Yu, Zhang, Priest, Reichle, \& Sheridan, 2018).

The experiment presented here examines how non-target (distractor) words are processed, testing the predictions of Reichle, Pollatsek, and Rayner's (2012) model of target word search. Note that within the much broader field of visual search (for a review see Eckstein, 2011) researchers have taken different approaches to accounting for eye movement behaviour during search, involving varying levels of visual and cognitive influences on oculomotor control (e.g. Torralba, Oliva, Castelhano, \& Henderson, 2006; Zelinksy, 2008). For example, within this broader field, studies have examined how eye movement behaviour may be modulated by similarities between the search target and distractors (e.g. Findlay, 1997). In contrast the present study focuses on eye movement behaviour during search in the absence of the target, and examines the effect of distractor characteristics that are unrelated to those of the target. Vanyukov, Warren, Wheeler and Reichle (2012) reported that the frequency of exposure to distractor stimuli modulated fixation durations on these non-linguistic stimuli during search. Vanyukov's work indicates that eye movement behaviour during search can be modulated by the visual 
familiarity of non-target stimuli. In contrast the present study examines the effect of the lexical characteristics of non-target (distractor) words during search within text.

In order to provide a test of whether the lexical characteristics of non-target words modulate eye movement control during target word search in Chinese, the word frequency of a critical word within each experimental sentence was manipulated ${ }^{1}$. For comparison, the study also provides a test of word frequency effects during reading for comprehension. It was anticipated that eye movement control during reading for comprehension in Chinese would be modulated by word frequency, in line with many previous studies in both alphabetic languages and in Chinese (e.g. Inhoff, \& Rayner, 1986; Yan, Tian, Bai, \& Rayner, 2006). However previous work indicates that eye movement behaviour for nontarget words during target word search in English is not modulated by word frequency (Rayner \& Fischer, 1996; Rayner \& Raney, 1996). Post-hoc analyses undertaken by Yu et al. (2018) also showed no effects of character frequency on eye movement behaviour during search for Chinese characters (though there were effects of character visual complexity). Therefore it was predicted that, in contrast to reading for comprehension, word frequency would not modulate eye movement behaviour for non-target words during target word search in Chinese. Together such findings would indicate that for both alphabetic and logographic languages, eye movement control for non-target words during target word search is driven at least largely by only superficial orthographic processing of the text. That is, there may be similarity in the mechanisms underlying the process of target word search across languages (see Liversedge et al., 2016). Such a pattern would be in line with the predictions of Reichle, Pollatsek, and Rayner's (2012) model of target word search. Reichle et al.'s (2012) model is an adaptation of the E-Z Reader model 
(Reichle, Warren, \& McConnell, 2009) but with adjustments to the parameters such that there is minimal lexical processing and no post-lexical processing of non-target words during target word search.

A further aim of the current study was to undertake detailed analyses to provide insights into the control of fixation durations and the prevalence of word skipping of nontarget words during search for target words in Chinese. The distribution of fixation durations was examined by fitting the ex-Gaussian distribution (Ratcliff, 1979; Staub, White, Drieghe, Hollway, \& Rayner, 2010) to single fixation durations. Shorter fixation durations on non-target words during target word search compared to reading might be accounted for by a leftward shift in the location of the distribution (Normal component, $\mu$ ) and/or an effect on the slow tail of the distribution (exponential component, $\tau$ ). It has been suggested that manipulations that affect $\tau$ (the skew of the distribution) may be indicative of processing difficulty (Staub \& Benatar, 2013). Given that processing of non-target words during target word search should require only superficial orthographic analysis, and therefore incur very little processing difficulty, it was anticipated that the skew of the distribution would be minimal during search compared to reading. Previous work in English (Rayner \& Raney, 1996; Rayner \& Fischer, 1996) indicates that fixation durations are shorter during target word search compared to reading, that there are higher skipping rates and that saccade lengths are longer. Consequently there is likely to be very limited time for parafoveal preview during target word search, and previews of words are likely to be more visually degraded compared to during reading for comprehension. Crucially, if there are high skipping rates and short fixation durations for non-target words during target word search in Chinese, this would indicate that rapid processing of visually 
degraded parafoveal text is central to understanding the mechanisms underlying the process of target word search across languages.

\section{Method}

Participants. Forty eight students (18-25yrs) from Liaoning Normal University participated in the experiment for course credit. All participants were native Chinese speakers with normal or corrected-to-normal vision, no history of dyslexia and all were naive to the purpose of the experiment.

Apparatus. An SR Research Eyelink 1000 eye tracker was used to record eye movements with a sampling rate of $1000 \mathrm{~Hz}$. Viewing was binocular, but only the right eye was recorded. Sentences were presented as single lines of text in black Song font on a light grey background, displayed on a Dell P190sb monitor. The viewing distance was $70 \mathrm{~cm}$ and one character subtended approximately 0.8 degrees of visual angle.

Materials and design. The design of the experiment was 2 X 2 (Task [reading, target word search] $\times$ Critical word frequency [high, low]), within participants and items. In total there were 30 practice sentences, 72 experimental items and 36 filler sentences. Filler items always included the search target word “zebra" (斑马). 斑马 is a transparent compound word, the first character corresponds to the meaning for stripe, and the second to horse. The search target word "zebra", a monomorphemic word in English, was also used in the study employed by Rayner and Fischer (1996). Across the filler items the target appeared equally often in the first third, middle third, and end third of each filler sentence. Critical high and low frequency two-character words were embedded in the experimental item sentence frames as shown in Table 1 (experimental and filler items are 
available as a Supplemental file). The four conditions were manipulated within participants and items following a Latin square design. Each participant completed two blocks, a reading block and a target word search block, the order of the blocks was counterbalanced. Within each block, the order of the experimental and filler items was randomised for each participant. For the reading block, $26 \%$ of sentences were followed by a comprehension question.

Insert Table 1 here

Word frequencies (in counts per million) were calculated using the SUBTLEX-CH corpus (Cai \& Brysbaert, 2010). High frequency critical words were significantly more frequent $(M=165, S D=233)$ than low frequency critical words $(M=1.9, S D=2.5)$ $(t(142)=5.96, p<.01)$. There was no difference in character visual complexity (total number of strokes) between the high $(M=13.5, S D=2.9)$ and low $(M=13.4, S D=3.3)$ frequency critical word conditions $(t<1)$. To ensure that the critical words were equally predictable across the two conditions twelve additional participants undertook a cloze task; they were presented with the beginning portions of the sentences up until the critical word and they attempted to guess which word might occur next. None of the critical words were guessed, demonstrating that neither the high nor low frequency critical words were predictable based on the prior sentence context.

Procedure. A chin-rest and forehead rest minimized head movements. The eye tracker was calibrated using a 3-point horizontal calibration. The calibration was checked centrally before every trial and at the three calibration positions every three trials, ensuring 
that calibration accuracy was less than 0.3 degrees at each of the three calibration positions. Re-calibrations were undertaken if necessary. Participants had to accurately fixate on a fixation cross at the position of the start of the line of text before each item was presented. In the reading block participants pressed a button to indicate when they had finished reading each sentence; they also used buttons to respond yes or no to comprehension questions. In the search block participants used buttons to respond present / absent once their search was complete for each sentence. At the end of the experiment (similar to the procedure of Rayner \& Fischer, 1996) participants were given a list of 40 sentences, of which ten appeared in the reading block, ten appeared in the search block, plus 20 filler items that had not appeared in either block. Participants indicated which sentences they recognized as having been presented in the experiment.

Analyses. Fixations shorter than $80 \mathrm{~ms}$ and longer than $1200 \mathrm{~ms}$ were removed (2.1\% of fixations). Sentence-level measures include response (read / search) time, average fixation duration, number of fixations, proportion of words skipped during firstpass (proportion of words that did not receive a first-pass fixation), proportion of words skipped entirely (proportion of words that were never fixated), average progressive saccade length and the overall number of inter-word regressions (total number of backwards eye movements to words earlier in the sentence). For analyses of the critical word, trials were excluded if there were blinks before or after first-pass fixation on the critical word (3.5\% of trials). Critical word measures include the proportion of word skips (proportion of critical words that did not receive a first-pass fixation), first-fixation duration (duration of the first-fixation on the critical word during first-pass), gaze duration (the sum of first-pass fixations on the critical word before leaving it) and total time (sum 
of all the fixations on the critical word). Note that the pattern for single fixation durations was very similar to that for first-fixation durations, and so for brevity is omitted here. Researchers can contact the authors to gain access to the data.

Linear mixed effects models (LMMs, Baayen, Davidson, \& Bates, 2008) were undertaken using R (R Core Team, 2016) and the lme4 package (Bates, Mächler, \& Bolker, 2011). Participants and items were specified as crossed-random effects. For continuous measures maximal random effects structures were used (following Barr, Levy, Scheepers \& Tily, 2013). For continuous variables analyses were undertaken for both untransformed and log-transformed data. However for transparency only results for untransformed data are reported as these two sets of analyses produced the same patterns of results. For dichotomous variables generalized linear mixed models were employed. The standard significance criterion for $t / z$ values for a two-tailed test (corresponding to $p<$ $0.05)$ is $>1.96$. Significance criteria were corrected for multiple comparisons within each family of tests, details are provided within Table notes (see von der Malsburg \& Angele, 2017).

Sentence-level measures were analysed using LMMs with task (read vs. search) as a fixed effect, defined using sliding contrasts in the MASS package (Venables \& Ripley, 2002). Given previous research, for the critical words we anticipated that there would be significant effects of word frequency during reading for comprehension (Yan et al., 2006), but no effects of word frequency during target word search (Rayner \& Fischer, 1996; Rayner \& Raney, 1996; see also Yu et al., 2018). In order to test directly for effects of word frequency during reading and during search, a full set of contrasts were undertaken. The contrasts tested whether there were effects of word frequency (high vs. low) for each 
level of task (reading and searching). These contrasts were coded for each task with an inverse contrast matrix (with the high frequency condition coded as -.5 and the low frequency condition coded as .5). Importantly, the LMM analyses cannot provide evidence for the null hypothesis, that there is no effect of word frequency during target word search (see Gallistel, 2009). In order to examine the strength of the evidence Bayes factors were calculated. For each of the temporal measures for the critical word (firstfixation duration, gaze duration, total time), for each task, models including a contrast for word frequency (high vs. low) were compared to intercept only models. Bayes factors were calculated in R (R Core Team, 2016) using the $\mathrm{lmBF}$ function in the BayesFactor package (Morey \& Rouder, 2015). Participants and items were specified as random effects. The scaling factor for g-priors was set at 0.5, with 100,000 Monte Carlo iterations.

In order to better understand the effect of task on when the eyes move, the distribution of fixation durations across the experimental items were examined by fitting the observed data to the ex-Gaussian distribution (Ratcliff, 1979). In order to maximise the comparability of the fixations across tasks, these analyses were undertaken only for single fixation durations (refixations, regressions and re-reading fixations are much less likely to occur during target word search). Also, in order to reflect fixation durations during the main reading/search process, first and final fixations for each trial were also excluded (final fixations in particular may be affected by the response requirement in the search task). The ex-Gaussian distribution is the convolution of a Normal and an exponential distribution. The parameters $\mu$ and $\sigma$ represent the location (mean) and standard deviation respectively of the Normal component. The $\tau$ parameter characterises 
the exponential component. Distributions were fit for each participant and each condition, and therefore a large number of observations were required. Analyses were based on the data from 46 participants, two other participants were removed due to fewer than 30 observations in one of the conditions. The best-fitting parameters were determined using the QMPE software (Cousineau, Brown, \& Heathcote, 2004; Heathcote, Brown, \& Mewhort, 2002), as described in Staub et al. (2010).

\section{Results}

Response accuracy was very high both to comprehension questions in the reading task $(M=94 \%, S D=5.7)$ and when indicating target presence or absence in the search task $(M=98 \%, S D=2.4)$. For the post-test, participants correctly categorized sentences as being present or absent during the experiment for $90 \%$ of sentences that were presented in the reading block $(S D=11.3$; range $60-100 \%)$ compared to $54 \%$ of sentences that were presented in the target word search block $(S D=25.8$, range $0-100 \%)$. The percentage of correctly categorized sentences was significantly higher in the reading compared to the search condition $(t(47)=10.25, p<.001)$, and performance in the search condition was no better than chance $(50 \%)(t<1)$. These results are consistent with those of Rayner and Fischer (1996) and are in line with the argument that text is processed much more superficially during target word search compared to reading (though note that there is considerable variability in sentence recognition accuracy across participants for the target word search task; it could be that some participants process text more superficially than others during target word search). 
Sentence-level measures. Means and standard errors for sentence-level measures are shown in Tables 2 and 3 for experimental and filler items respectively. These tables also include the LMM statistics for effects of task. For both experimental and filler items, for search compared to reading for comprehension, response times were shorter, there were fewer fixations, longer progressive saccades and fewer inter-word regressions. For the filler trials, average fixation durations were longer during target word search, which could be due to longer fixation durations associated with identification of the presence of the search target word, as well as time associated with the "present" response decision. For the experimental trials, there was no overall effect of task on average fixation duration, though further exploration of the data revealed that, for target word search, first and last fixations tended to be longer than other fixations in the trial (see below for further analyses of single fixation durations).

Insert Tables 2 and 3 here

Perhaps the most striking finding from the sentence-level measures are the high levels of word skipping during target word search, $\sim 60 \%$ of words were skipped entirely (compared to $\sim 25 \%$ during reading). To examine word skipping in more detail additional analyses were undertaken for the experimental items. Figure 1 shows the proportion of progressive interword saccades that fixate the following word $(n+1)$ or that skip one or multiple consecutive words. The Figure illustrates that often during target word search at least two consecutive words are skipped (destination $n+3$ or beyond), whereas this occurs much more rarely during reading for comprehension. Skipping rates were further 
examined for one and two character words (the most common word lengths in Chinese), excluding the first and last words within each sentence. These analyses confirmed that words were more likely to be skipped during first-pass for target word search compared to reading for both one (Reading: $M=0.63, S D=0.48$; Search: $M=0.83, S D=0.38$ ) and two (Reading $M=0.20, S D=0.40 ;$ Search $M=0.62, S D=0.49)$ character words $(z \mathrm{~s}>$ 21). The same pattern held for words skipped entirely for both one (Reading: $M=0.51$., $S D=0.50$; Search: $M=0.80, S D=0.40$ ) and two (Reading: $M=0.12 ., S D=0.32$; Search: $M=0.55, S D=0.50)$ character words $(z \mathrm{~s}>29)$. Together these analyses clearly demonstrate that processing of non-target words during target word search in Chinese is often based on parafoveal preview (or postview) rather than direct fixation of words.

Insert Figure 1 here

Further analyses of word skipping were undertaken for the filler trials to examine if the search target can be correctly identified as "present" even if it is not directly fixated (analyses limited to trials with correct responses for the search task). Again, the proportion of target words skipped was much higher during search compared with reading for comprehension, both during first-pass (reading: $M=0.20, S D=0.40$; search: $M=0.43$, $S D=0.49$ ) and across the entire trial (reading: $M=0.14, S D=0.34$; search: $M=0.27, S D$ $=0.44)(z \mathrm{~s}>7)$. The results indicate that the search target word may be correctly identified as present based on a parafoveal preview or postview, that is, without it being directly fixated. To summarise, the results highlight that target word search in Chinese can be characterized by very high levels of word skipping, indicating that parafoveal 
processing is likely to be especially important when searching for words of interest in Chinese text.

In order to examine in more detail how task modulated fixation durations during the main part of the trial (excluding first and last fixations) for the experimental items, the distribution of single fixation durations were examined by fitting the ex-Gaussian distribution. The mean best fitting ex-Gaussian parameters, and the results of the paired samples $t$-tests for each parameter, are shown in Table 4. All three of the ex-Gaussian parameters were significantly smaller during target word search compared to reading. These results are in line with the pattern shown in the Vincentile plot (Ratcliff, 1979; Vincent, 1912) (Figure 2). The points on the plot represent the mean observed data for each participant within each of the bins (first bin includes the shortest $10 \%$ of fixations, second bin includes the next shortest $10 \%$ etc.). The triangles represent predicted values based on the ex-Gaussian parameters (see Figure caption). Crucially the pattern for the observed data shown in the plot is consistent with the differences in the ex-Gaussian parameters. The distribution of single fixation durations is located $(\mu)$ further to the left during target word search compared to reading, in line with clear separation of the curves across all of the Vincentile bins. There is also more variability during reading compared to search for target words as indicated by the standard deviation of the normal component $(\sigma)$. Single fixation durations also show a reduced degree of skew $(\tau)$ during target word search compared to reading, in line with the greater separation of the curves for the higher Vincentiles. Together these results demonstrate that, compared to reading for comprehension, during the main period of search single fixations of all durations are shorter during search, the durations are more consistent and there are relatively few 
especially long durations. This pattern is consistent with the argument that the influence of text characteristics on the control of fixation durations is limited during target word search. The short duration of the fixations is also particularly striking given the long progressive saccades (and likelihood of skipping consecutive words). Not only can target word search apparently be very dependent on parafoveal processing of words, it appears that during the main part of the search process there is often relatively little time to undertake processing of this visually degraded parafoveal input.

Insert Table 4 and Figure 2 here

Critical word measures. Means and standard errors for the critical word measures for the experimental items are shown in Table 5. Planned LMM contrasts for effects of word frequency (high vs. low) for each task ${ }^{3}$ are also detailed in Table 5. In line with previous research (Yan et al., 2006) reading times were longer on low compared to high frequency words during reading. There were no significant effects of word frequency on the likelihood of word skipping.

Insert Table 5 here

Consistent with the null hypothesis, the contrasts for reading times on the critical word during target word search revealed no effects of word frequency ${ }^{4}$. In order to examine the strength of the evidence for the null effects of word frequency on reading times during target word search, Bayes factors (BF) were calculated (reported in Table 5). 
For the reading task the BFs were all greater than 10, which we interpret as providing strong support (in line with Vandekerckhove, Matzke, \& Wagenmakers, 2015; based on Jeffreys, 1961) for an effect of word frequency during reading. However for target word search, all of the BFs were less than one which we interpret as providing moderate evidence (in line with Vandekerckhove et al., 2015) in favour of the intercept only model. The Bayes factors therefore indicate a strong effect of word frequency during reading, and moderate evidence that there is no effect of word frequency on non-target word reading times during target word search.

\section{Discussion}

The present study indicates that the lexical characteristics of non-target words do not ordinarily modulate eye movement behaviour during target word search in Chinese. Furthermore, high levels of word skipping during search indicate that processing of visually degraded parafoveal text may be especially important when searching for words in Chinese text. The implications for understanding the nature of text processing and eye movement control during target word search are explored below.

In line with previous experimental work on target word search in English text (Rayner \& Fischer, 1996; Rayner \& Raney, 1996) the present study showed no effects of word frequency on eye movement measures for non-target words during target word search in Chinese. Together the results provide no evidence for an effect of lexical processing on eye movement behaviour for non-target words during target word search in text for two very different orthographies (alphabetic and logographic). The results also fit with Yu et al.'s (2018) post-hoc analyses which showed no effect of character frequency 
during search for Chinese characters. However it has been suggested that there may be occasional lexical influences on fixation durations during target word search (Reichle et al., 2012). The conventional approaches employed so far are unlikely to reveal such occasional influences on behaviour. However it is possible that distributional analyses, undertaken in future studies with very large numbers of items (see Footnote 4), may possibly reveal these. If there were any lexical influences of non-target words on fixation durations during target word search it is quite likely that such influences would occur late during a fixation. Parafoveal previews are likely to be especially eccentric and therefore visually degraded during target word search (due to long saccade lengths). Consequently parafoveal previews during target word search are unlikely to provide an opportunity for a "head start" in any lexical processing of subsequently fixated words (see Reichle \& Reingold, 2013; Reingold, Reichle, Glaholt, \& Sheridan, 2012). Therefore any occasional lexical influences on fixation durations for non-target words during target word search are likely to occur late during a fixation. Future work may therefore examine whether word frequency may possibly have a small influence on the skew of the distribution of fixation durations on non-target words during target word search.

Importantly, as Rayner and Raney (1996) noted, the absence of an influence of word frequency on eye movement behaviour during target word search does not necessarily imply that lexical processing does not occur for non-target words during search for a word in text. For fixated words at least, lexical access may still occur automatically (MacLeod, 1991; but see Kiefer \& Martens, 2010; Kinoshita, De Wit, \& Norris, 2017) but, as described in the previous paragraph, it may only rarely modulate control of eye movements. It could be that other methods, such as Event Related 
Potentials (Dampure, Benraiss, \& Vibert, 2016), perhaps with co-registration of eye movements (Dimigen, Sommer, Hohfield, Jacobs, \& Kliegl, 2011), may help reveal whether lexical processing does indeed take place during target word search within text. Other manipulations, such as effects of context, may also help clarify if non-target words are processed at a linguistic level during target word search (Fisher, 1975; Lefton \& Fisher, 1976).

The results of the present study are in line with the notion that only a superficial level of orthographic processing ordinarily drives eye movement control for non-target words during target word search in text (Reichle et al., 2012). It could be that attention is shifted from one word to the next during target word search, similar to shifts of attention during reading (see Reichle et al., 2012). Alternatively it could be that simple visual and oculomotor processes might largely drive eye movement control (e.g. O’Regan, 1992), or there may be a deadline for when saccade programming must be initiated (Henderson $\&$ Ferreira, 1990). Mechanisms underlying search for target words may also have similarities with those employed during search for non-linguistic stimuli (e.g. Zelinsky, 2008). For example, in contrast to reading for comprehension, during target word search text may be processed that is below the current fixated line of text (Pollatsek et al., 1993). Further work comparing target word search across a range of text formats (see Lefton, 1979) may help reveal to what extent the mechanisms underlying search for target words in text may employ the same eye movement control routines developed for reading meaningful and normally formatted text (as in the simulations reported by Reichle et al., 2012). 
Overall the present study highlights that, compared to eye movement behaviour during reading, eye movement behaviour during target word search in Chinese provides limited time for visual sampling of the text, and non-target words are often sampled only in visually degraded parafoveal vision. Despite these limitations, the short duration foveal and parafoveal inputs appear to provide sufficient time, and sufficient visual detail, to undertake at least the superficial orthographic processing of words necessary to accurately locate a target word in Chinese text. Further work in this area may reveal in more detail the role of parafoveal preview during target word search. For example, identifying the region of text that can be processed in order to determine the presence of the search target, as well as the area of text from which useful parafoveal preview can be extracted (Rayner \& Fisher, 1987).

It would also be valuable for further research to examine, for both alphabetic and logographic languages, the factors that modulate eye movement behaviour during target word search, for example, the effect of target and distractor word characteristics (Dampure et al., 2016; Léger et al., 2012; Shen \& Reingold, 2001; Smith, Lott, \& Cronnell, 1969; Spragins et al., 1976). Research with non-linguistic stimuli indicates that eye movement behaviour is affected by the presence of distractors sharing physical characteristics with the target (Findlay, 1997). Similar effects may occur during search for target words in text. For example, Chinese characters or radicals which are visually similar to those within a target word may be more likely to be fixated during search (e.g. more fixations on 班, when the search target is 斑马). Parafoveal preview may also be important in determining any such effects of target-distractor similarity on fixation durations (Reingold \& Glaholt, 2014). Other studies may examine how readers respond to successfully 
locating a target word, and how behaviour might be modulated by the characteristics of the target word. Variables such as the number of times target words are encountered or the number of search target words may also modulate search behaviour. Furthermore, an important area of study for future research is the issue of how readers switch from the task of locating relevant text to comprehending it (see Simola, Salojärvi, \& Kojo, 2008). Finally, the present study examined behaviour during search for a single repeated target word within a series of individual unrelated sentences. As noted in the Introduction, understanding the mechanisms that underlie how we identify text relevant to our reading goals is especially important now that so much textual information is available to us. Consequently it will also be important to examine whether the mechanisms underlying search for target words within single sentences also hold within much longer texts. 
Acknowledgements

The work was supported by an International Exchanges Scheme grant from the Royal Society (IE141063). We thank Simon Liversedge, Raymond Bertram and Bernhard Angele, as well as anonymous reviewers, for their helpful comments on an earlier version of the manuscript. 
References

Baayen, R.H., Davidson, D.J., \& Bates, D.M. (2008). Mixed-effects modeling with crossed random effects for subjects and items. Journal of Memory and Language, $59,390-412$.

Barr, D.J., Levy, R., Scheepers, C., \& Tily, H.J. (2013). Random effects structure for confirmatory hypothesis testing: Keep it maximal. Journal of Memory and Language, 68, 255-278.

Bates, D., Mächler, M., \& Bolker, B.M. (2011). LME4: linear mixed-effects models using S4 classes. R Package Version 0.999375-39. http://CRAN.Rproject.org/package $=\operatorname{lme} 4$

Brysbaert, M., \& Stevens, M. (2018). Power analysis and effect size in mixed effects models: A tutorial. Journal of Cognition, 1:9, 1-20.

Cai, Q., \& Brysbaert, M. (2010). SUBTLEX-CH: Chinese word and character frequencies based on film subtitles. PloS ONE, 5, 1-8.

Cauchard, F., Eyrolle, H., Cellier, J.-M., \& Hyona, J. (2010). Visual signals vertically extend the perceptual span in searching a text: A gaze-contingent window study. Discourse Processes, 47, 617-640.

Cousineau, D., Brown, S., \& Heathcote, A. (2004). Fitting distributions using maximum likelihood: Methods and packages. Behaviour Research Methods, Instruments, \& Computers, 36, 742-756.

Dampure, J., Benraiss, A., \& Vibert, N. (2016). Task-dependent modulation of word processing mechanisms during modified visual search tasks. The Quarterly Journal of Experimental Psychology, 69, 1145-1163. 
Dampuré, J., Ros, C., Rouet, J-F., \& Vibert, N. (2012). How word familiarity facilitates visual search for verbal material. Applied Cognitive Psychology, 26, 271-288.

Dimigen, O., Sommer, W., Hohfield, A., Jacobs, A.M., \& Kliegl, R. (2011).

Coregistration of eye movements and EEG in natural reading: analyses and review. Journal of Experimental Psychology: General, 140, 552-572.

Eckstein, M.P. (2011). Visual search: A retrospective. Journal of Vision, 11, 14.

Findlay, J.M. (1997). Saccade target selection during visual search. Vision Research, 37, $617-631$.

Fisher, D.F. (1975). Reading and visual search. Memory \& Cognition, 3, 188-196.

Gallistel, C.R. (2009). The importance of proving the null. Psychological Review, 116, $439-453$

Goonetilleke, R.S., Lau, W.C., \& Smith, H.M. (2002). Visual search strategies and eye movements when searching Chinese character screens. International Journal of Human-Computer Studies, 57, 447-468.

Grabbe, J.W. (2014). Effects of targets embedded within words in a visual search task. Advances in Cognitive Psychology, 10, 1-8.

Heathcote, A., Brown, S., \& Mewhort, D. J. K. (2002). Quantile maximum likelihood estimation of response time distributions. Psychonomic Bulletin \& Review, 9, 394401.

Henderson, J.M., \& Ferreira, F. (1990). Effects of foveal processing difficulty on the perceptual span in reading: Implications for attention and eye movement control. Journal of Experimental Psychology: Learning, Memory and Cognition, 16, 417429. 
Inhoff, A.W., \& Rayner, K. (1986). Parafoveal word processing during eye fixations in reading: Effects of word frequency. Perception \& Psychophysics, 40, 431-439.

Jeffreys, H. (1961). Theory of Probability (3rd Ed.). Oxford UK: Oxford University Press.

Kaakinen, J.K., Hyönä, J., \& Keenan, J.M. (2002). Perspective effects on online text processing. Discourse Processes, 33, 159-173.

Kiefer, M., \& Martens, U. (2010). Attentional sensitization of unconscious cognition: Task sets modulate subsequent masked semantic priming. Journal of Experimental Psychology: General, 139, 464-489.

Kinoshita, S., De Wit, B., \& Norris, D. (2017). The magic of words reconsidered: Investigating the automaticity of reading color-neutral words in the Stroop task. Journal of Experimental Psychology: Learning, Memory and Cognition, 43, 369384.

Klusewitz, M. A., \& Lorch, R. F., Jr. (2000). Effects of headings and familiarity with a text on strategies for searching a text. Memory \& Cognition, 28, 667-676.

Lefton, L.A. (1979). Reading and searching through geometrically transformed text. In: Kolers P.A., Wrolstad M.E., Bouma H. (eds) Processing of Visible Language. Nato Conference Series, Vol 13. Springer, Boston, MA.

Lefton, L.A., \& Fisher, D.F. (1976). Information extraction during visual search: A developmental progression. Journal of Experimental Child Psychology, 22, 346361.

Léger, L., Rouet, J-F., Ros, C., \& Vibert, N. (2012). Orthographic versus semantic matching in visual search for words within lists. Canadian Journal of Experimental Psychology, 66, 32-43. 
Liversedge, S.P., Drieghe, D., Li, X., Yan, G., Bai, X., \& Hyönä, J. (2016). Universality in eye movements and reading: A trilingual investigation. Cognition, 147, 1-20.

Liu, Z. (2005). Reading behavior in the digital environment: Changes in reading behavior over the past ten years. Journal of Documentation, 61, 700-712.

MacLeod, C. M. (1991). Half a century of research on the Stroop effect: An integrative review. Psychological Bulletin, 109, 163-203.

Morey, R.D., \& Rouder, J.N. (2015). BayesFactor: Computation of Bayes factors for common designs. $\mathrm{R}$ package version 0.9-12-2.

Ojanpää, H., Näsänen, R., \& Kojo, I. (2002). Eye movements in the visual search of word lists. Vision Research, 42, 1499-1512.

O’Regan, J.K. (1992). Optimal viewing position in words and the strategy-tactics theory of eye movements in reading. In K. Rayner (Ed.), Eye movements and visual cognition: Scene perception and reading (pp. 333-354). New York: SpringerValag.

Pollatsek, A., Raney, G.E., LaGasse, L., \& Rayner, K. (1993). The use of information below fixation in reading and in visual search. Canadian Journal of Experimental Psychology, 47, 179-200.

R Core Team (2016). R: A language and environment for statistical computing. $\mathrm{R}$ Foundation for Statistical Computing, Vienna, Austria. https://www.R-project.org/.

Ratcliff, R. (1979). Group reaction time distributions and an analysis of distribution statistics. Psychological Bulletin, 86, 446-461.

Rayner, K. (2009). Eye movements and attention in reading, scene perception, and visual search. Quarterly Journal of Experimental Psychology, 62, 1457-1506. 
Rayner, K., \& Fisher, D.L. (1987). Letter processing during eye fixations in visual search. Perception \& Psychophysics, 42, 87-100.

Rayner, K., \& Fischer, M. H. (1996). Mindless reading revisited: Eye movements during reading and scanning are different. Perception \& Psychophysics, 58, 734-747.

Rayner, K., Li, X., Williams, C.C. Cave, K.R., \& Well, A.D. (2007). Eye movements during information processing tasks: Individual differences and cultural effects. Vision Research, 47, 2714-2726.

Rayner, K., \& Raney, G. E. (1996). Eye movement control in reading and visual search: Effects of word frequency. Psychonomic Bulletin \& Review, 3, 245-248.

Reichle, E. D., Pollatsek, A., \& Rayner, K. (2012). Using E-Z Reader to simulate eye movements in nonreading tasks: A unified framework for understanding the eyemind link. Psychological Review, 119, 155-185.

Reichle, E.D., \& Reingold, E.M. (2013). Neurophysiological constraints on the eye-mind link. Frontiers in Human Neuroscience, 7, 361.

Reingold, E. M., Reichle, E. D., Glaholt, M. G., \& Sheridan, H. (2012). Direct lexical control of eye movements in reading: Evidence from a survival analysis of fixation durations. Cognitive Psychology, 65, 177-206.

Reichle, E.D., Warren, T., \& McConnell, K. (2009). Using E-Z Reader to model the effects of higher level language processing on eye movements during reading. Psychonomic Bulletin \& Review, 16, 1-21.

Reingold, E.M., \& Glaholt, M.G. (2014). Cognitive control of fixation duration in visual search: The role of extrafoveal processing. Visual Cognition, 22, 3-4.

Scialfa, C.T., Caird, J.K., Connolly, K., \& Cosmescu, C. (1998). Effects of APA reference 
format on search performance and preference. Journal of Experimental Psychology: Applied, 4, 44-54.

Shen, J., \& Reingold, E.M. (2001). Visual search asymmetry: The influence of stimulus familiarity and low-level features. Perception \& Psychophysics, 63, 464-475.

Simola, J., Salojärvi, J., \& Kojo, I. (2008). Using hidden Markov model to uncover processing states from eye movements in information search tasks. Cognitive Systems Research, 9, 237-251.

Smith, F., Lott, D., \& Cronnell, B. (1969). The effect of type size and case alternation on word identification. The American Journal of Psychology, 82, 248-253.

Spragins, A.B., Lefton, L.A., \& Fisher, D.F. (1976). Eye movements while reading and searching spatially transformed text: A developmental examination. Memory \& Cognition, 4, 36-42.

Staub, A., \& Benatar, A. (2013). Individual differences in fixation duration distributions in reading. Psychonomic Bulletin \& Review, 20, 1304-1311.

Staub, A., White, S.J., Drieghe, D., Hollway, E.C., \& Rayner, K. (2010). Distributional effects of word frequency on eye fixation durations. Journal of Experimental Psychology: Human Perception and Performance, 36, 1280-1293.

Torralba, A., Oliva, A., Castelhano, M.S., \& Henderson, J.M. (2006). Contextual guidance of eye movements and attention in real-world scenes: The role of global features in object search. Psychological Review, 113, 766-786.

Vandekerckhove, J., Matzke, D., \& Wagenmakers, E.-J. (2015). Model comparison and the principle of parsimony. In J. Busemeyer, J. Townsend, Z. J. Wang, \& A. Eidels (Eds.), Oxford Handbook of Computational and Mathematical Psychology, (pp. 300- 
319). Oxford: Oxford University Press.

Vanyukov, P.M., Warren, T., Wheeler, M.E., \& Reichle, E.D. (2012). The emergence of frequency effects in eye movements. Cognition, 123, 185-189.

Venables, W.N., \& Ripley, B.D. (2002). Modern applied statistics with $S$ (4th ed.). New York, USA: Springer.

Vincent, S. B. (1912). The function of the viborissae in the behaviour of the white rat (Animal Behaviour Monographs Vol. 1, No. 5). New York, NY: H. Holt.

von der Malsburg, T., \& Angele, B. (2017). False positives and other statistical errors in standard analyses of eye movements in reading. Journal of Memory and Language, 94, 119-133.

White, S.J., Warrington, K.L., McGowan, V.A., \& Paterson, K.B. (2015). Eye movements during reading and topic scanning: Effects of word frequency. Journal of Experimental Psychology: Human Perception and Performance, 41, 233-248.

Yan, G., Tian, H., Bai, X., \& Rayner, K. (2006). The effect of word and character frequency on the eye movements of Chinese readers. British Journal of Psychology, 97, 259-68.

Yu, L., Zhang, Q., Priest, C., Reichle, E.D., \& Sheridan, H. (2018). Charactercomplexity effects in Chinese reading and visual search: A comparison and theoretical implications. Quarterly Journal of Experimental Psychology, 71, 140151.

Zang, C., Liversedge, S.P., Bai, X., \& Yan, G. (2011). Eye movements during Chinese reading. In, I. Gilchrist \& S. Everling (Eds.), The Oxford Handbook of Eye Movements (pp. 961-978). Oxford, UK: Oxford University Press. 
Zelinsky, G.J. (2008). A theory of eye movements during target acquisition. Psychological Review, 115, 787-835.

Zhou, W., Mo, F., Zhang, Y., \& Ding, J., (2017). Semantic and syntactic associations during word search modulate the relationship between attention and subsequent memory. The Journal of General Psychology, 144, 69-88. 


\section{Footnotes}

${ }^{1}$ Note that the present study employed the same search target word throughout. Future work may examine the different issue of whether the word frequency of the target word itself might modulate search behaviour for both target and non-target words (see: Dampuré et al., 2012; Grabbe, 2014).

${ }^{2}$ In Chinese, "zebra" is an infrequent word (3.28 counts per million, SUBTLEX$\mathrm{CH})$, the first character is composed of twelve strokes and the second character is composed of three strokes. The characteristics of any search target word may affect participants' search behaviour. For example, search may have been facilitated by the visually distinctive and infrequent letter " $z$ " in Rayner and Fischer's (1996) study, or by the visually simple second character of the target word in the present study. Given Yu et al.'s (2018) findings, skipping rates during target word search may be lower than reported here if the target word characters are more visually complex.

${ }^{3}$ For completeness, for the critical word measures, LMM models including task (read vs. search), word frequency (high vs. low) and the interaction were also undertaken. All four measures produced clear effects of task $(t / z>4.4)$. The $t / z$ values for the interaction term were as follows: Skipping $z=2.10$; first-fixation duration $t=-1.05$; gaze duration $t=-2.45$; total time $t=-2.73$.

${ }^{4}$ The experiment was designed such that there were 18 items per participant per condition. However due to skipping and data exclusions on average each participant contributed $\sim 7$ data points for each high and low frequency condition for target word search, and $~ 14$ data points for each high and low frequency condition for reading. 
Brysbaert and Stevens (2018) recommend a total of at least 1,600 observations per condition. Therefore, in order to provide a very strong test of whether there are effects of word frequency during target word search, given high skipping rates, a larger number of items would be necessary. Ideally future studies would employ sufficient items such that all participants would be likely to generate enough fixations on the critical word per condition to enable examination of any effects of word frequency on the distribution of fixation durations (see Discussion). 
Figure captions

Figure 1. Experimental items. For each task, proportion of progressive inter-word saccades moving to the following word (destination $n+1$ ), skipping one word (destination $\mathrm{n}+2$ ), or skipping multiple consecutive words (destination $n+3, n+4$, etc).

Figure 2. Vincentile plot for single fixation durations during reading and search for a target word. Error bars represent the standard error of the mean. The plot also shows the predicted Vincentiles, generated from 50,000 samples on the basis of the best-fitting exGaussian parameters. 
Table 1. Example Experimental Item and Filler Item with English Translations. Critical Words and Search Target Shown in Bold.

Sentence type Critical word frequency Sentence

Experimental High 专家们对这个计划的价值进行了评估。

The value of the plan was evaluated by experts.

Low 专家们对这个屏风的价值进行了评估。

The value of the screen was evaluated by experts. 猎人的一声枪响把斑马惊得向四处逃窝。

The hunter's gunshot frightened zebra into fleeing in all directions. 
Table 2. Experimental Items: Mean Sentence-Level Measures During Reading and Target Word Search. Standard Errors Shown in Parentheses. Linear Mixed Effect Model Statistics for the Effect of Task for Each Measure (Read vs. Search).

\begin{tabular}{|c|c|c|c|c|c|}
\hline \multirow[t]{2}{*}{ Sentence-Level Measures } & \multirow[t]{2}{*}{ Read } & \multirow[t]{2}{*}{ Search } & \multicolumn{3}{|l|}{ LMM } \\
\hline & & & $\beta$ & $S E$ & $\mathrm{t}$ \\
\hline Response time (ms) & $3183(35.4)$ & $807(5.9)$ & -2376.71 & 155.83 & $-15.25 *$ \\
\hline Average fixation duration (ms) & $288(1.22)$ & $288(1.59)$ & -0.63 & 4.39 & -0.14 \\
\hline Number of fixations & $11.5(0.11)$ & $4.1(0.02)$ & -7.41 & 0.44 & $-16.78 *$ \\
\hline Words skipped on first-pass (proportion) & $0.34(0.003)$ & $0.66(0.002)$ & 0.32 & 0.01 & $23.81 *$ \\
\hline Words skipped entirely (proportion) & $0.26(0.003)$ & $0.61(0.002)$ & 0.35 & 0.01 & $29.65^{*}$ \\
\hline Progressive saccade length (characters) & $2.65(0.02)$ & $5.43(0.04)$ & 2.78 & 0.13 & $21.27 *$ \\
\hline Number of inter-word regressions & $1.86(0.04)$ & $0.55(0.02)$ & -1.31 & 0.15 & $-8.70 *$ \\
\hline
\end{tabular}

Note: * Denotes statistical significance $(t>2.69$, corrected for the seven measures examined in this Table). 
Table 3. Filler Items: Mean Sentence-Level Measures During Reading and Target Word Search. Standard Errors Shown in Parentheses. Linear Mixed Effect Model Statistics for the Effect of Task for Each Measure (Read vs. Search).

\begin{tabular}{|c|c|c|c|c|c|}
\hline \multirow[t]{2}{*}{ Sentence-Level Measures } & \multirow[t]{2}{*}{ Read } & \multirow[t]{2}{*}{ Search } & \multicolumn{3}{|l|}{ LMM } \\
\hline & & & $\beta$ & $S E$ & $\mathrm{t}$ \\
\hline Response time (ms) & $3111(50.0)$ & $777(18.6)$ & -2333.51 & 172.30 & $-13.54 *$ \\
\hline Average fixation duration (ms) & $288(1.70)$ & $316(2.86)$ & 28.21 & 6.12 & $4.61 *$ \\
\hline Number of fixations & $11.4(0.15)$ & $3.8(0.05)$ & -7.58 & 0.50 & $-15.07 *$ \\
\hline Words skipped on first-pass (proportion) & $0.32(0.005)$ & $0.68(0.004)$ & 0.36 & 0.02 & $18.78^{*}$ \\
\hline Words skipped entirely (proportion) & $0.24(0.005)$ & $0.64(0.004)$ & 0.39 & 0.02 & $22.04 *$ \\
\hline Progressive saccade length (characters) & $2.65(0.03)$ & $4.91(0.06)$ & 2.26 & 0.13 & $17.87 *$ \\
\hline Number of inter-word regressions & $1.79(0.06)$ & $0.44(0.02)$ & -1.35 & 0.17 & $-7.86^{*}$ \\
\hline
\end{tabular}

Note: * Denotes statistical significance $(t>2.69$, corrected for the seven measures examined in this Table). 
Table 4. Mean Best Fitting Ex-Gaussian Parameters for Single Fixation Durations (Excluding First and Last Fixations) in the Reading and Target Word Search Tasks.

\begin{tabular}{llll}
\hline Ex-Gaussian & \multicolumn{2}{l}{ Task } & \multirow{2}{*}{$t$ value } \\
\cline { 2 - 3 } parameter & Reading & Target word search & \\
\hline$\mu$ & 176 & 153 & $6.39 *$ \\
$\sigma$ & 35 & 23 & $4.99 *$ \\
$\tau$ & 88 & 38 & $12.15^{*}$ \\
\hline
\end{tabular}

Note: The degrees of freedom for each of the $t$-tests was 45.

* Denotes statistical significance, corrected for 3 comparisons $(p<.016)$. 
Table 5. Mean Critical Word Measures for the Experimental Items During Reading and Target Word Search for High and Low Frequency Words (Standard Errors Shown in Parentheses). Linear Mixed Effect Model Statistics for High vs. Low Frequency

Contrasts for Each Task. Bayes Factors for Reading Time Measures (Models Including a Contrast for Word Frequency Compared with Intercept Only Models).

\begin{tabular}{|c|c|c|c|c|c|c|c|}
\hline \multicolumn{2}{|c|}{ Critical word measures } & \multicolumn{2}{|l|}{ Means } & \multicolumn{3}{|c|}{ LMM contrast } & \multirow[t]{2}{*}{ Bayes Factor } \\
\hline & & High frequency & Low frequency & $b$ & se & $t / z$ & \\
\hline \multirow[t]{4}{*}{ Reading } & Skipping (first-pass) (proportion) & $0.20(0.01)$ & $0.16(0.01)$ & -0.22 & 0.13 & -1.66 & - \\
\hline & First-fixation duration (ms) & $272(4.2)$ & $292(4.5)$ & 19.23 & 6.50 & $2.96^{*}$ & 10.32 \\
\hline & Gaze duration (ms) & $300(5.6)$ & $351(6.7)$ & 48.97 & 9.65 & $5.08 *$ & 843095 \\
\hline & Total time (ms) & $421(10.1)$ & $499(13.3)$ & 75.60 & 20.62 & $3.67 *$ & 2812 \\
\hline Target & Skipping (first-pass) (proportion) & $0.61(0.02)$ & $0.64(0.02)$ & 0.13 & 0.10 & 1.29 & - \\
\hline \multirow[t]{3}{*}{ word search } & First-fixation duration (ms) & $233(7.0)$ & $242(6.9)$ & 7.61 & 8.58 & 0.89 & 0.13 \\
\hline & Gaze duration (ms) & $234(7.1)$ & $249(7.5)$ & 13.85 & 10.88 & 1.27 & 0.24 \\
\hline & Total time (ms) & $257(7.3)$ & $272(7.2)$ & 14.19 & 16.62 & 0.85 & 0.21 \\
\hline
\end{tabular}

Note: * Denotes statistical significance $(t / z>2.50$, corrected for the four measures examined in this Table). 
Figure 1. Experimental items. For each task, proportion of progressive inter-word saccades moving to the following word (destination $n+1$ ), skipping one word (destination $\mathrm{n}+2$ ), or skipping multiple consecutive words (destination $n+3, n+4$, etc).

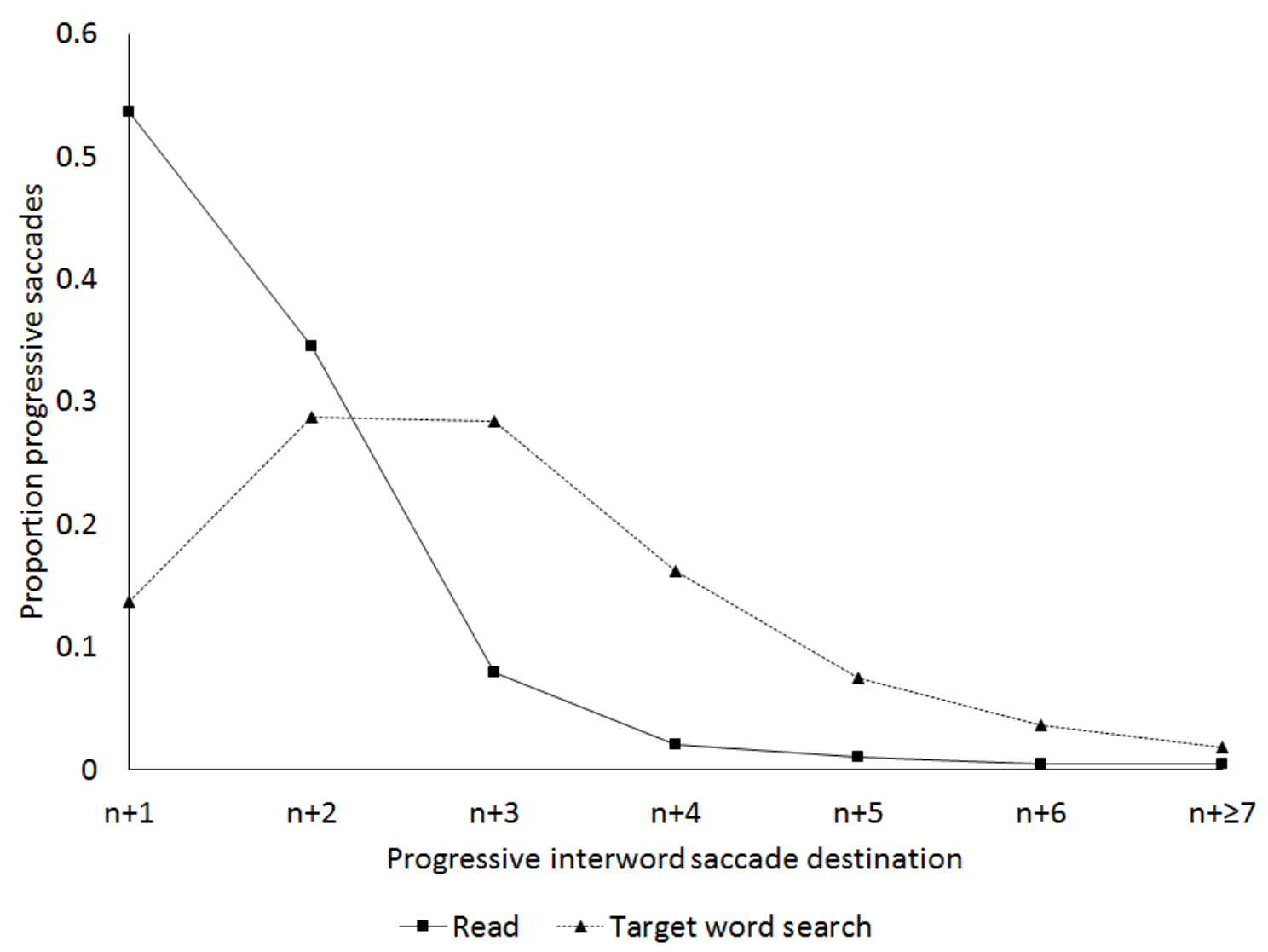


Figure 2. Vincentile plot for single fixation durations during reading and search for a target word. Error bars represent the standard error of the mean. The plot also shows the predicted Vincentiles, generated from 50,000 samples on the basis of the best-fitting exGaussian parameters.

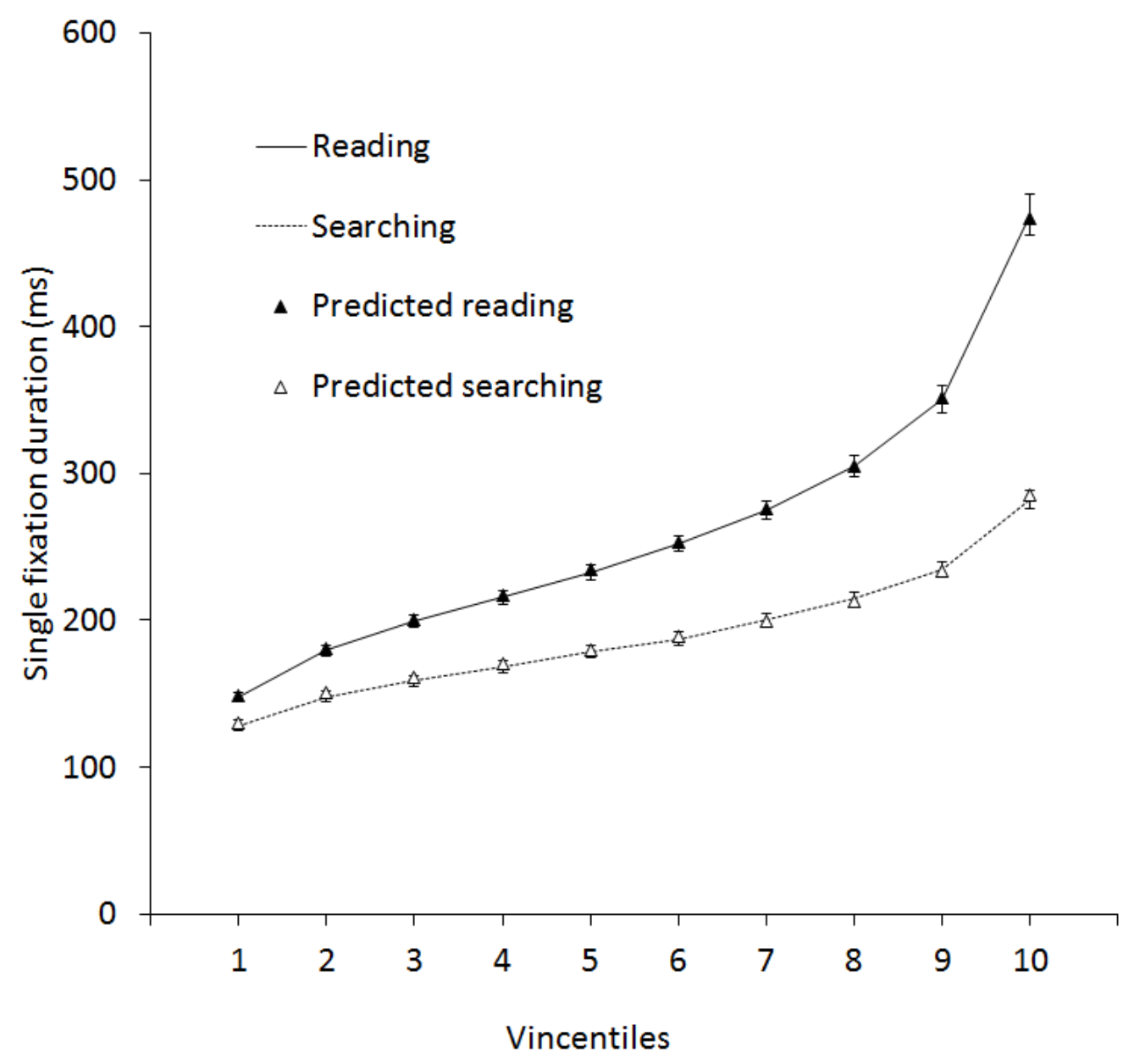

